\title{
Cholangiocarcinomas can originate from hepatocytes in mice
}

\author{
Biao Fan, ${ }^{1,2}$ Yann Malato, ${ }^{3,4}$ Diego F. Calvisi, ${ }^{5}$ Syed Naqvi, ${ }^{3}$ Nataliya Razumilava, ${ }^{6}$ Silvia Ribback, ${ }^{5}$ \\ Gregory J. Gores, ${ }^{6}$ Frank Dombrowski, ${ }^{5}$ Matthias Evert, ${ }^{5}$ Xin Chen, ${ }^{1,7}$ and Holger Willenbring ${ }^{3,4,7}$
}

\begin{abstract}
1Department of Bioengineering and Therapeutic Sciences, UCSF, San Francisco, California, USA. 2Department of Surgery, Beijing Cancer Hospital and Institute, Peking University School of Oncology and Key Laboratory of Carcinogenesis and Translational Research (Ministry of Education), Beijing, China. ${ }^{3}$ Eli and Edythe Broad Center of Regeneration Medicine and Stem Cell Research and ${ }^{4}$ Department of Surgery, Division of Transplantation, UCSF, San Francisco, California, USA. IInstitut für Pathologie, Ernst-Moritz-Arndt-Universität, Greifswald, Germany. ${ }^{6}$ Division of Gastroenterology and Hepatology, Mayo Clinic, Rochester, Minnesota, USA. ${ }^{2}$ Liver Center, UCSF, San Francisco, California, USA.
\end{abstract}

\begin{abstract}
Intrahepatic cholangiocarcinomas (ICCs) are primary liver tumors with a poor prognosis. The development of effective therapies has been hampered by a limited understanding of the biology of ICCs. Although ICCs exhibit heterogeneity in location, histology, and marker expression, they are currently thought to derive invariably from the cells lining the bile ducts, biliary epithelial cells (BECs), or liver progenitor cells (LPCs). Despite lack of experimental evidence establishing BECs or LPCs as the origin of ICCs, other liver cell types have not been considered. Here we show that ICCs can originate from fully differentiated hepatocytes. Using a mouse model of hepatocyte fate tracing, we found that activated $\mathrm{NOTCH}$ and AKT signaling cooperate to convert normal hepatocytes into biliary cells that act as precursors of rapidly progressing, lethal ICCs. Our findings suggest a previously overlooked mechanism of human ICC formation that may be targetable for anti-ICC therapy.
\end{abstract}

\section{Introduction}

Cholangiocarcinomas (CCs) are adenocarcinomas that can be distinguished by their anatomic location. Extrahepatic CCs may cause symptoms such as jaundice and are therefore occasionally detected at resectable stages (1). In contrast, intrahepatic CCs (ICCs) long remain asymptomatic and are therefore typically diagnosed at therapy-resistant advanced stages. The resulting poor prognosis and increasing incidence of ICCs necessitate a better understanding of their pathogenesis.

ICCs frequently grow alongside or into the lumen of bile ducts and are thought to originate from biliary epithelial cells (BECs). The heterogeneous histology of ICCs suggests that they can derive from both cylindrical BECs lining large bile ducts (ductal ICCs) or cuboidal BECs lining small bile ducts or ductules (peripheral ICCs) (2). Ductules also contain liver progenitor cells (LPCs), which can give rise to both BECs and hepatocytes and are also being considered as an origin of ICCs, particularly of rare tumors such as cholangiolocellular carcinomas that exhibit both ICC and hepatocellular carcinoma (HCC) characteristics.

An alternative mechanism explaining the formation of ICC could be lineage conversion occurring during malignant transformation of hepatocytes. To our knowledge, this possibility has so far not been considered. However, the common developmental origin of BECs and hepatocytes, and the previous finding that forced activation of NOTCH signaling can convert hepatocytes into cells resembling BECs in mice, provide indirect support for this concept (3). The clinical observation that peripheral ICCs can form masses in the liver parenchyma, where mainly hepatocytes reside, is also compatible with a hepatocyte origin (4). Furthermore, ICCs can be caused not only by diseases affecting BECs, such as parasitic infections, primary sclerosing cholangitis, bile

Authorship note: Biao Fan, Yann Malato, and Diego F. Calvisi contributed equally to this work.

Conflict of interest: The authors have declared that no conflict of interest exists. Citation for this article: J Clin Invest. 2012;122(8):2911-2915. doi:10.1172/JCI63212. duct cysts, and hepatolithiasis, but also by diseases that cause hepatocyte injury, such as hepatitis $\mathrm{C}$ or B infection, alcohol abuse, and nonalcoholic steatohepatitis (5).

The first direct evidence in support of a hepatocyte origin of ICCs was provided by our previous finding that hydrodynamic tail vein injection of a human AKT overexpression plasmid into mice produced mainly HCCs, but also a small number of cholangiocellular lesions (6). However, although this technique delivers plasmids mainly to hepatocytes in the liver (7), we could not rule out plasmid entry and AKT overexpression in BECs or LPCs, especially because AKT was under the control of a ubiquitous promoter. Thus, a hepatocyte origin of the cholangiocellular lesions could not be definitely established. In addition, our finding of only benign tumors, but not ICCs, suggested that overexpression of AKT alone is not sufficient for ICC formation.

Here, we hypothesized that activated NOTCH signaling can convert hepatocytes into ICC precursors because of its ability to induce biliary differentiation of hepatocytes in mice (3) and its emerging role as a driver and prognostic marker in human CCs $(8,9)$. Indeed, using our previously reported hepatocyte fate-tracing model (10), we found that activated NOTCH signaling, not alone, but in combination with AKT overexpression, causes rapid formation of ICCs from hepatocytes.

\section{Results and Discussion}

To activate NOTCH signaling in the liver, we stably overexpressed the intracellular domain of the NOTCH1 receptor (NICD; Myc-tagged). For this purpose, we delivered plasmids for sleeping beauty transposase-mediated genomic transgene integration to livers of wild-type FVB/N mice by hydrodynamic tail vein injection (6). We did not detect histological changes within 10 weeks after NICD plasmid injection (data not shown). By 20 weeks, we found cystic cholangiocellular tumors resembling human biliary cystadenomas (Supplemental Figure 1, A and B). Some of these tumors contained cytologically malignant cells that not only expanded intracystically, but also invaded the sur- 


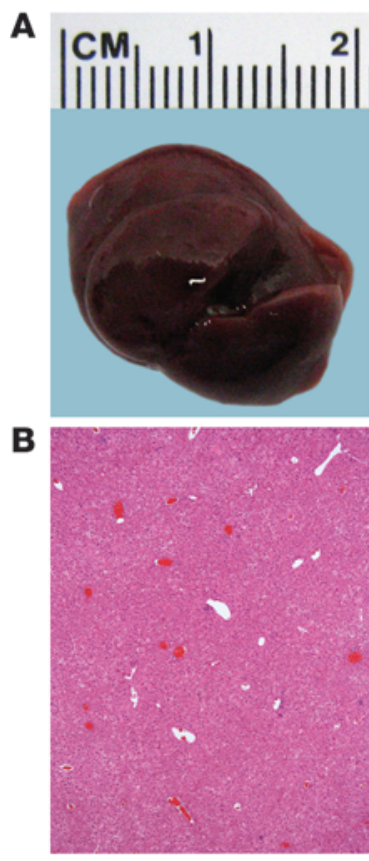

$1.5 \mathrm{wk}$
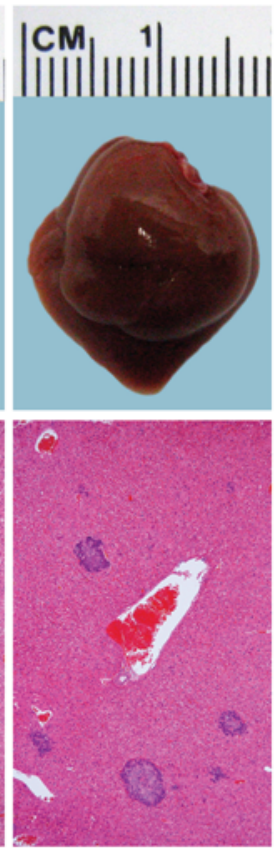

2.5 wk
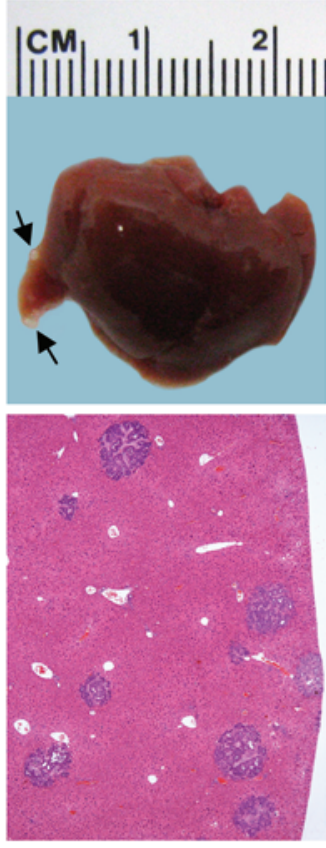

$3.5 \mathrm{wk}$
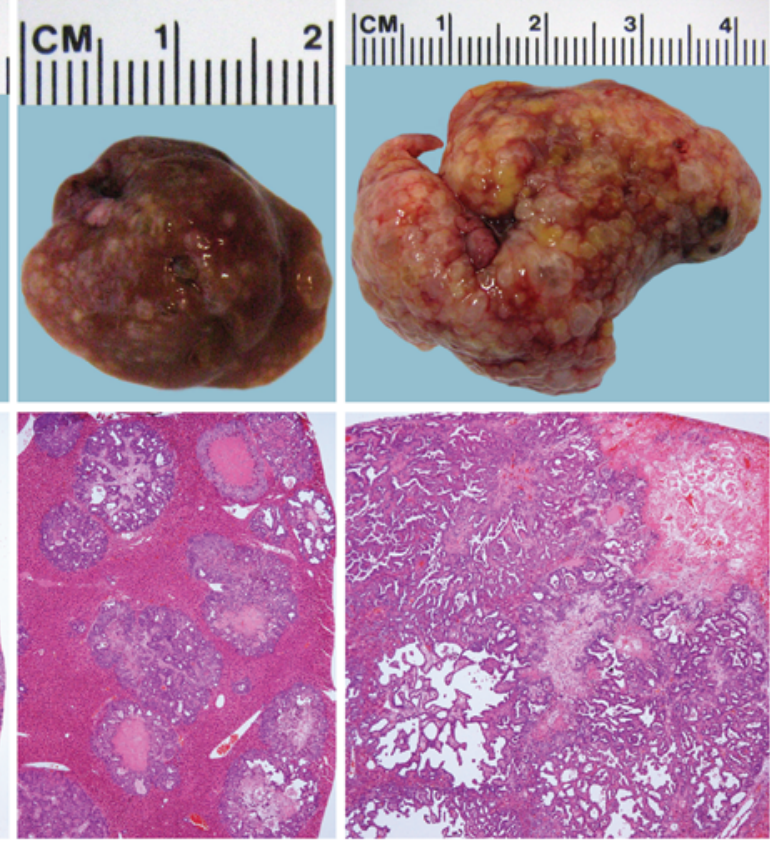

$4.5 \mathrm{wk}$

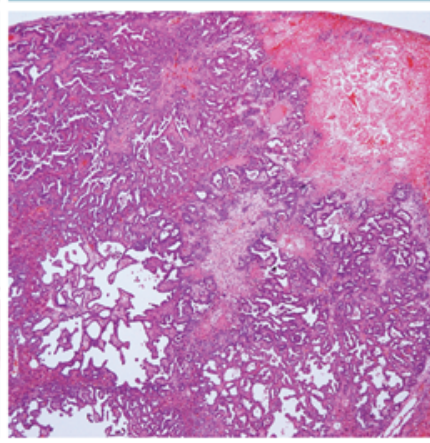

$5 \mathrm{wk}$

Figure 1

NICD/AKT-induced ICC formation. (A) Photographic images of mouse livers taken at different time points after NICD/AKT plasmid injection. Small tumors (arrows) were visible as early as 3.5 weeks after injection. (B) H\&E stainings of corresponding liver sections show rapidly expanding tumors replacing the normal liver parenchyma. Original magnification, $\times 40$. At least 15 liver sections from 3 mice were analyzed for each time point.

rounding liver tissue (Supplemental Figure 1, C-E), consistent with progression to invasive cystadenocarcinomas. All tumors were derived from cells that stably overexpressed NICD (Supplemental Figure 1F).

Considering the rapid onset of hepatocyte proliferation and formation of premalignant lesions in mice overexpressing AKT in the liver (6), we decided to inject the NICD plasmid together with an AKT overexpression plasmid (HA-tagged; combination referred to as NICD/AKT) into mice. Macroscopically, livers of these mice appeared normal 1.5 and 2.5 weeks after injection (Figure 1A). However, small, white, cyst-like lesions were present on the liver surface after 3.5 weeks. These lesions rapidly expanded and by 4.5 weeks occupied most of the liver surface. At 5 weeks after plasmid injection, the lesions had replaced most of the normal liver tissue, and the mice rapidly deteriorated and either died or needed to be euthanized (Supplemental Figure 2).

At the microscopic level, single or clusters of cytologically malignant cells could be identified 1.5 weeks after plasmid injection (Figure 1B and Supplemental Figure 3, A and B). These clusters progressed into small tumors of ductular phenotype by 2.5 weeks (Supplemental Figure 3, C and D). The tumors grew markedly by 3.5 weeks and exhibited either a ductular or cystic phenotype (Supplemental Figure 3, E and F). Although cytologically malignant, many tumors still had well-defined borders at this stage. By 4.5 weeks, however, most tumors showed additional signs of malignancy, including necrosis, high mitotic activity, and invasion of the surrounding liver parenchyma (Supplemental Figure 3, G and $\mathrm{H}$ ), characteristics that correspond to human ICCs. Immunostaining and immunoblotting for the Myc and HA tags showed that tumors invariably derived from cells stably overexpressing NICD/AKT (Supplemental Figure 4, A and B). Additional immunostainings for the biliary marker cytokeratin 19 (Ck19) and major urinary protein (Mup), a marker specific for hepatocytes (10), confirmed that the tumors exhibited exclusively biliary differentiation (Supplemental Figure 5, A and B), providing further support for their classification as ICCs. Along these lines, expression of $A f p$, a gene overexpressed in HCCs, remained at low levels, whereas expression of Epcam, a gene specific for biliary cells in the liver, markedly increased with time after injection (Supplemental Figure 6, A and B). Thus, overexpression of NICD/AKT in the liver induces specifically ICCs.

Many ICCs formed in the central area of the liver lobule, where normally hepatocytes, but not BECs or LPCs, reside (Supplemental Figure 3B). Therefore, we hypothesized that the NICD/AKTinduced ICCs originated from hepatocytes. To rule out migrant BECs or LPCs as the origin, we used our previously reported hepatocyte fate-tracing model, in which all hepatocytes and their progeny, but no other liver cells, express enhanced yellow fluorescent protein (EYFP) (10). To generate the model, we intravenously injected $4 \times 10^{11}$ viral genomes of a double-stranded adenoassociated viral vector serotype 8 expressing Cre recombinase from the hepatocyte-specific transthyretin promoter (AAV8-Ttr-Cre) into mice that carry EYFP disrupted by a floxed stop codon in the ubiquitously expressed Rosa26 locus (R26R-EYFP mice) (Figure 2A). To replicate the experiments described above, we used FVB/N R26R-EYFP mice. We analyzed a subset of the mice 1 week after injection to ascertain that AAV8-Ttr-Cre looped out the stop codon and activated EYFP expression in hepatocytes with the 
A

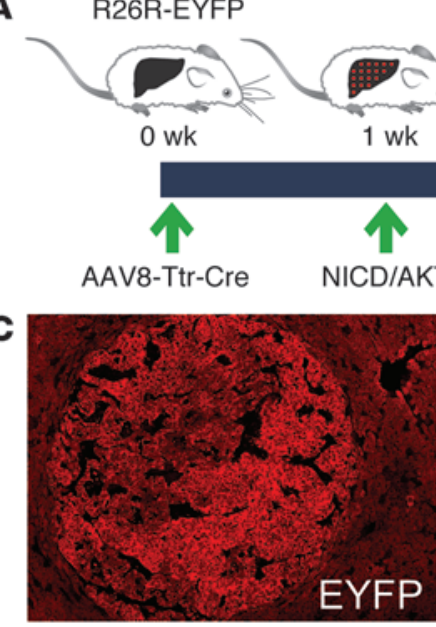

D

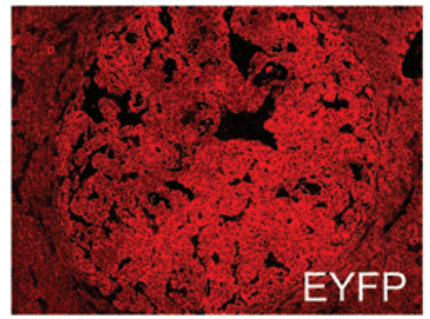

E

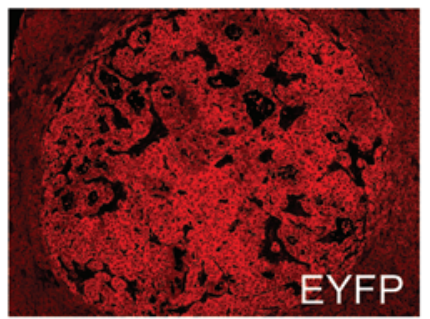

B

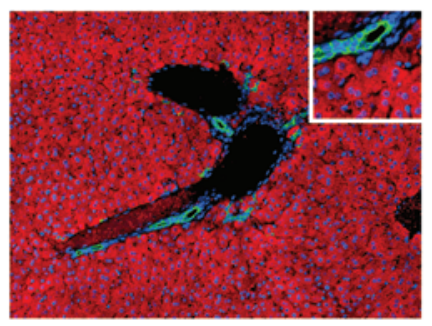

Liver analysis
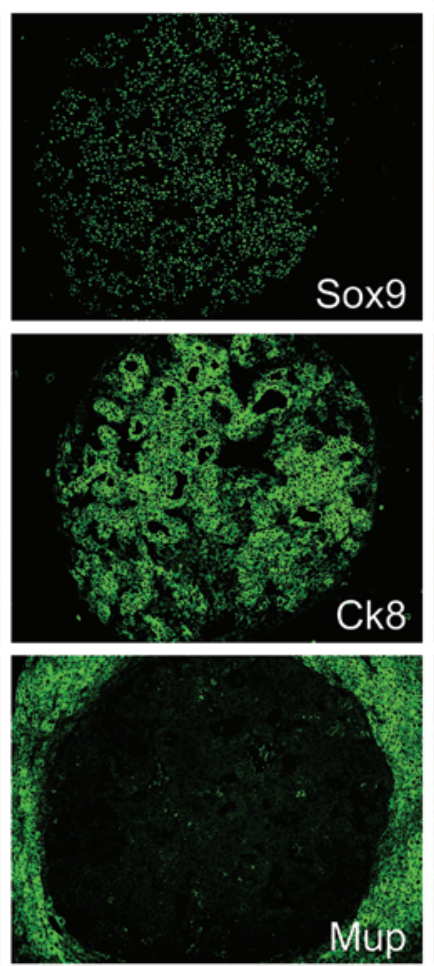
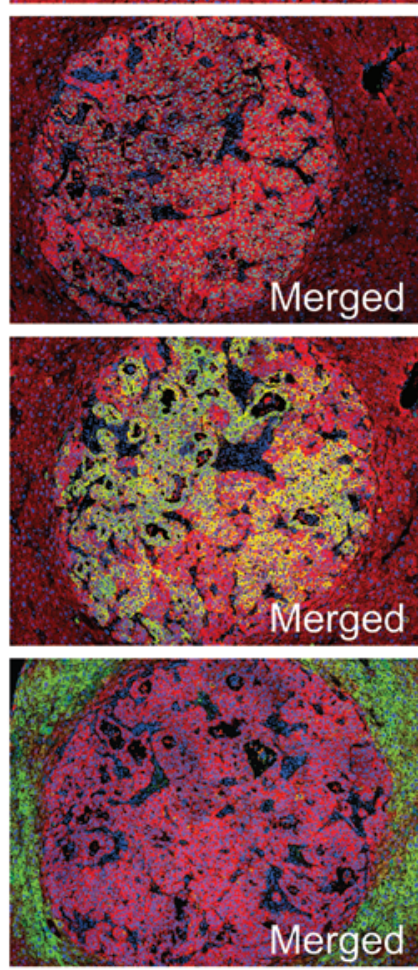

\section{Figure 2}

Hepatocyte origin of NICD/ AKT-induced ICCs. (A) R26REYFP mice were intravenously injected with $4 \times 10^{11}$ viral genomes of AAV8-Ttr-Cre, followed by hydrodynamic tail vein injection of the NICD/AKT plasmids 1 week later. Tumors were analyzed 4.5 weeks after plasmid injection. (B) Coimmunostaining for EYFP (red) and $\mathrm{Ck} 19$ (green) 1 week after AAV8-Ttr-Cre injection shows that all hepatocytes, but no BECs, express EYFP. (C-E) Immunostainings of tumors for EYFP (red) show that they originated from hepatocytes. Additional immunostainings (all green) for Sox9 (C), Ck8 (D), and Mup (E) show that tumors express biliary markers but lack hepatocyte differentiation. Nuclei were stained with DAPI (blue). Original magnification, $\times 100$; inset, $\times 200$. At least 15 liver sections from 3 mice were analyzed for each immunostaining. same efficiency and specificity as previously reported (Figure $2 \mathrm{~B}$ and ref. 10). We hydrodynamically injected the remaining mice with the NICD/AKT plasmids.

As expected, all mice receiving the NICD/AKT plasmids harbored numerous large ICCs 4.5 weeks later. HA immunostaining showed that all ICCs originated from cells stably overexpressing NICD/AKT (Supplemental Figure 7A). Positive EYFP immunostaining revealed that the cells of origin of ICCs were hepatocytes (Figure 2, $\mathrm{C}-\mathrm{E}$ ). Confirming our results described above (Supplemental Figure 5, A and B), the ICCs expressed the biliary markers Sry-box containing gene 9 (Sox9), Ck8, and mucin 1 (Muc1), but were negative for the hepatocyte marker Mup (Figure 2, C-E, and Supplemental Figure 7B). Ck8 is expressed in mouse BECs from early developmental stages onward (Supplemental Figure 7C and ref. 11). The Muc1 protein is normally localized in the apical membrane of BECs (Supplemental Figure 7D and ref. 12). High or cytoplasmic expression of Muc1 is associated with progression and invasiveness of human ICCs $(13,14)$. Indeed, we observed intense, nonpolarized Muc1 labeling in many ICC cells (Supplemental Figure 7B). Furthermore, many cells in NICD/AKT-induced ICCs were positive for the proliferation marker Ki67 (Supplemental Figure $7 \mathrm{E}$ ), which is in accordance with what has been shown in human high-grade ICCs (15). To exclude that ICCs were EYFP positive because AAV8-Ttr-Cre became expressed in BECs or LPCs during malignant transformation, we injected AAV8-Ttr-Cre into R26REYFP mice as before, but eliminated this nonintegrating vector from the liver by $2 / 3$ partial hepatectomy before injection of the NICD/AKT plasmids (Supplemental Figure 8, A-C, and ref. 16). We found that ICCs remained EYFP positive in the absence of potential unspecific AAV8-Ttr-Cre expression (Supplemental Figure 8, D and E). These results show that hepatocytes give rise to bona fide ICCs in response to NICD/AKT overexpression.

To investigate the initial stages of NICD/AKT-induced ICC formation, we screened for single cells expressing NICD/AKT at 1.5 weeks after plasmid injection (Figure $3 \mathrm{~A}$ ). Although most cells expressing the NICD/AKT tags were located in the center of the liver lobule, they exhibited biliary differentiation, as was evident from expression of Ck8 (Supplemental Figure 9, A and B). Coimmunostaining for EYFP, Ck8, and Sox9 revealed that these cells were hepatocytes in which biliary gene expression had been activated (Figure 3B). Coimmunostaining for EYFP, Ck8, and the HA tag showed that all hybrid cells stably overexpressed NICD/AKT (Figure 3C). Interestingly, we failed to detect cells coexpressing EYFP, HA, and Ck19 at this time point. However, Ck19 expression was detectable at 2.5 weeks after plasmid injection, when single cells had formed small tumors (data not shown). Because Sox9 is also expressed in bipotential LPCs (17) and Ck8 is expressed in bile duct development at earlier stages than Ck19 (18), this find- 
A

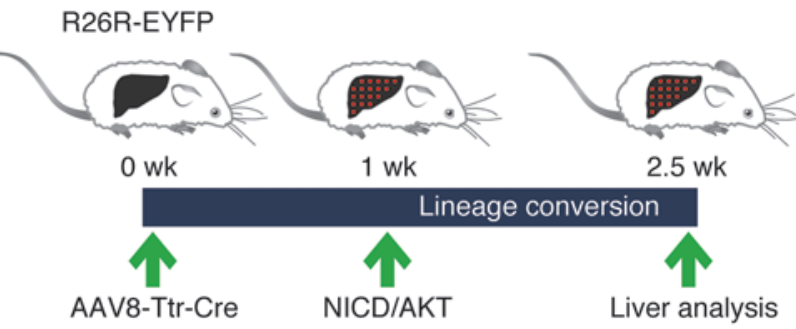

B
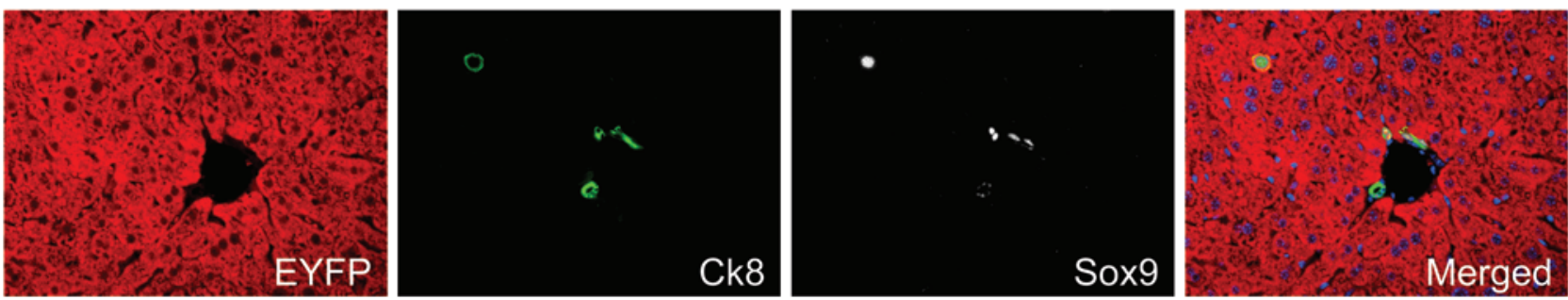

C
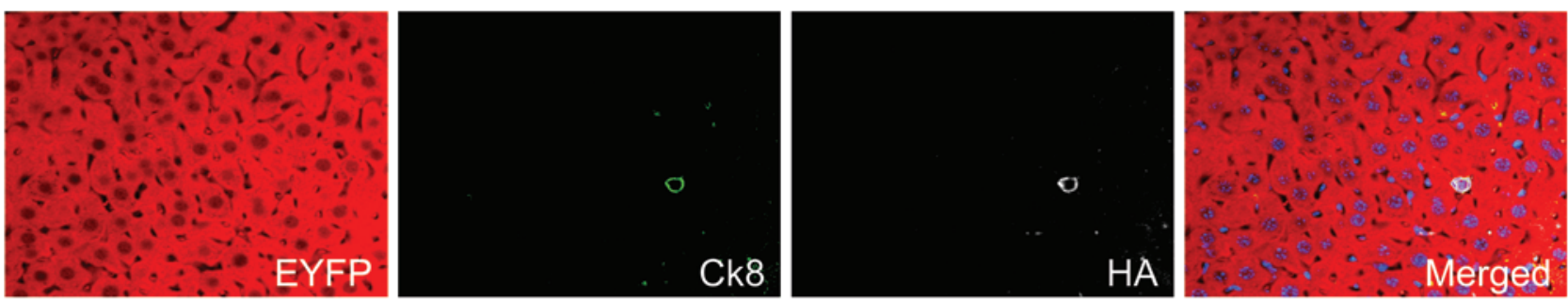

D
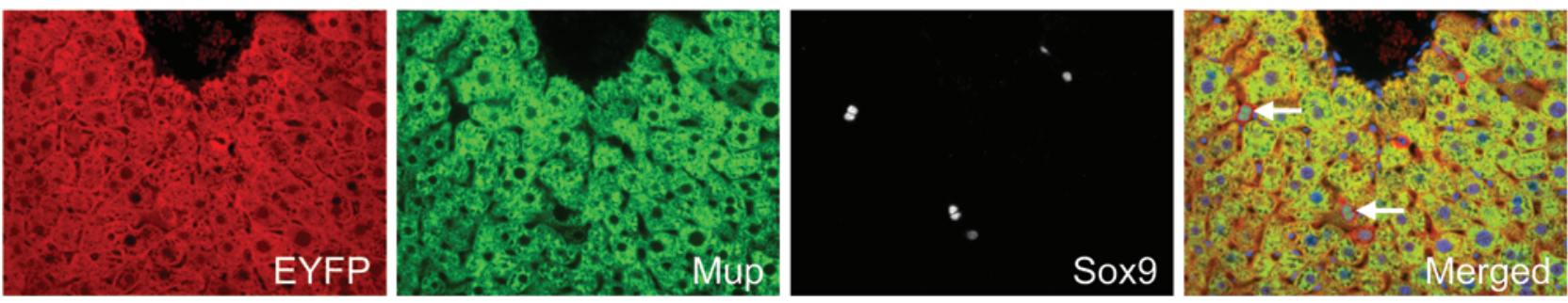

Figure 3

Conversion of hepatocytes into ICC precursors. (A) R26R-EYFP mice were intravenously injected with $4 \times 10^{11}$ viral genomes of AAV8-Ttr-Cre, followed by hydrodynamic tail vein injection of the NICD/AKT plasmids 1 week later. Livers were analyzed 1.5 weeks after plasmid injection. (B) Coimmunostaining for EYFP (red), Ck8 (green), and Sox9 (white) shows a hepatocyte expressing the early BEC and LPC markers Ck8 and Sox9. (C) Coimmunostaining for EYFP (red), Ck8 (green), and HA (white) shows that hybrid cells express the NICD/AKT plasmids. (D) Coimmunostaining for EYFP (red), Mup (green), and Sox9 (white) shows decreased Mup expression in hybrid cells. Original magnification, $\times 400$. At least 15 liver sections from 3 mice were analyzed for each immunostaining.

ing suggests that NICD/AKT-expressing hepatocytes gradually acquired biliary differentiation. In accordance with this assessment, we found that EYFP and Sox9 double-positive cells gradually lost hepatocyte differentiation, as indicated by declining levels of Mup, which is only expressed in mature hepatocytes (Figure 3D). Furthermore, electron microscopy revealed loss of glycogen stores in hepatocytes undergoing lineage conversion, and, illustrating their hepatocyte origin and difference from normal BECs, these cells showed formation of cell junctions and bile canaliculi with adjacent hepatocytes, lack of a basement membrane, and cytological atypia (Supplemental Figure 10, A-C). These findings show that NICD/AKT-induced conversion of hepatocytes into biliary cells occurs already at the single-cell stage and suggest concurrent initiation of malignant transformation.

In conclusion, our results show that overexpression of two factors, NICD and AKT, is sufficient for rapid conversion of fully differentiated, normal hepatocytes into lethal ICCs. Our finding that ICC formation involves conversion of hepatocytes into atypical biliary cells at an early, single-cell stage suggests that lineage reprogramming gives way to or promotes initiation of malignant transformation, which is then manifested by cell proliferation. Although the mechanism by which NICD and AKT cooperate to induce hepatocyte-derived ICCs remains to be determined, our finding that overexpression of NICD alone induces invasive cystadenocarcinomas suggests that it is the driving oncogene. AKT is likely acting to accelerate ICC formation by providing metabolic and thus proliferation-promoting support (6). In support of this assessment, overexpression of AKT alone produces mainly HCCs and a few benign cholangiocellular lesions (6), whereas overexpression of both NICD and AKT induced only ICCs in our model. NOTCH and AKT signaling are frequently coactivated in human ICCs (Supplemental Figure 11, A-C), which suggests that their cooperation is also driving human ICC formation. 
Whether ICCs originate from hepatocytes also in humans remains to be determined. However, this possibility is strongly suggested by recent findings of intracytoplasmic p $62^{+}$hyaline inclusions, which are thought to be specific for injured or malignantly transformed hepatocytes, in common peripheral ICCs (that is, not rare, potentially LPC-derived, cholangiolocellular carcinomas) (19). Most ICCs with $\mathrm{p} 62^{+}$hyaline inclusions emerged in patients with liver disease due to hepatitis $\mathrm{C}$ or $\mathrm{B}$ infection or alcohol abuse, which suggests that chronic hepatocyte injury caused lineage conversion and ICC formation. In accordance with these observations, epidemiologic studies have identified viral hepatitis as a risk factor for ICC (5). By establishing that hepatocytes can give rise to ICCs, our study sheds light on the pathogenesis of this cancer and suggests molecular targets for much-needed new therapies.

\section{Methods}

Supplemental Methods are available online with this article.

Mice. Six- to 10-week-old wild-type (Charles River) or R26R-EYFP (20) mice on the FVB/N strain background were used.

Plasmids. HA-tagged AKT (human AKT1) and hyperactive sleeping beauty transposase plasmids were described previously (6). AKT was myristoylated for constitutively active kinase activity. Mouse NOTCH1 receptor NICD sequence with Myc tag was obtained from Addgene. NICD and AKT were expressed from the EF1 $\alpha$ promoter. Transposase was expressed from the CMV promoter.

Hydrodynamic tail vein injection. Twenty micrograms of NICD, $4 \mu \mathrm{g}$ AKT, and $1 \mu \mathrm{g}$ transposase plasmids were diluted in $2 \mathrm{ml} 0.9 \% \mathrm{NaCl}$, sterile filtered, and injected into a lateral tail vein within 5-7 seconds as previously described (6).

AAV8-Ttr-Cre. Production, titering, and injection of AAV8-Ttr-Cre was performed as previously described (10).

Immunostaining. Liver samples were fixed overnight in zinc formalin (Anatech Ltd.), embedded in paraffin, cut into 5 - $\mu \mathrm{m}$-thick sections, and placed on Superfrost Plus slides (Fisher Scientif ic). Sections were deparaffinized and boiled in Antigen Retrieval Citra Solution (BioGenex) for 10 minutes. After blocking in $10 \%$ serum for 1 hour, sections were incubated with primary antibodies overnight at $4{ }^{\circ} \mathrm{C}$ and secondary antibodies for 1 hour at room temperature (Supplemental Tables 1 and 2). Nuclear DNA was stained with $300 \mathrm{nM}$ DAPI (Millipore).

Statistics. Data are expressed as mean \pm SEM. Statistical significance was determined by 2-way ANOVA followed by 2-tailed Student's $t$ test. A $P$ value less than 0.05 was considered significant.

Study approval. All mouse procedures were approved by the Institutional Animal Care and Use Committee at UCSF.

\section{Acknowledgments}

This work was supported by California Institute for Regenerative Medicine (CIRM) New Faculty Award RN2-00950 to H. Willenbring, NIH R01 CA136606 to X. Chen, Deutsche Forschungsgemeinschaft (DFG) Ev168/2-1 to M. Evert, DFG Do622/2-1 to F. Dombrowski, and P30 DK026743 (UCSF Liver Center). B. Fan was supported by the China Scholarship Council (contract 2010601079). The authors thank Lijie Jiang and Jason Au for technical assistance, Aras Mattis for discussion, and Pamela Derish for manuscript editing.

Received for publication February 3, 2012, and accepted in revised form June 1, 2012.

Address correspondence to: Xin Chen, UCSF, 513 Parnassus Avenue, Campus Box 0912, San Francisco, California 94143, USA. Phone: 415.502.6526; Fax: 415.502.4322; E-mail: chenx@pharmacy.ucsf.edu. Or to: Holger Willenbring, UCSF, 35 Medical Center Way, Campus Box 0665, San Francisco, California 94143, USA. Phone: 415.476.2417; Fax: 415.514.2346; E-mail: willenbringh@ stemcell.ucsf.edu.
1. Sempoux $C$, et al. Intrahepatic cholangiocarcinoma: new insights in pathology. Semin Liver Dis. 2011;31(1):49-60.

2. Komuta M, et al. Histological diversity in cholangiocellular carcinoma reflects the different cholangiocyte phenotypes. Hepatology. 2012;55(6):1876-1888.

3. Zong Y, et al. Notch signaling controls liver development by regulating biliary differentiation. Development. 2009;136(10):1727-1739.

4. Blechacz B, Komuta M, Roskams T, Gores GJ. Clinical diagnosis and staging of cholangiocarcinoma. Nat Rev Gastroenterol Hepatol. 2011;8(9):512-522.

5. Tyson GL, El-Serag HB. Risk factors for cholangiocarcinoma. Hepatology. 2011;54(1):173-184.

6. Calvisi DF, et al. Increased lipogenesis, induced by AKT-mTORC1-RPS6 signaling, promotes development of human hepatocellular carcinoma. Gastroenterology. 2011;140(3):1071-1083.

7. Zhang G, Song YK, Liu D. Long-term expression of human alpha1-antitrypsin gene in mouse liver achieved by intravenous administration of plasmid DNA using a hydrodynamics-based procedure. Gene Ther. 2000;7(15):1344-1349.

8. Yoon HA, et al. Clinicopathological significance of altered Notch signaling in extrahepatic cholan- giocarcinoma and gallbladder carcinoma. World J Gastroenterol. 2011;17(35):4023-4030.

9. Mazur PK, et al. Expression and clinicopathological significance of notch signaling and cell-fate genes in biliary tract cancer. Am J Gastroenterol. 2012;107(1):126-135

10. Malato Y, et al. Fate tracing of mature hepatocytes in mouse liver homeostasis and regeneration. J Clin Invest. 2011;121(12):4850-4860.

11. Hofmann JJ, Zovein AC, Koh H, Radtke F, Weinmaster G, Iruela-Arispe ML. Jagged1 in the portal vein mesenchyme regulates intrahepatic bile duct development: insights into Alagille syndrome. Development. 2010;137(23):4061-4072.

12. Raynaud P, et al. A classification of ductal plate malformations based on distinct pathogenic mechanisms of biliary dysmorphogenesis. Hepatology. 2011;53(6):1959-1966.

13. Sasaki M, Nakanuma Y, Kim YS. Characterization of apomucin expression in intrahepatic cholangiocarcinomas and their precursor lesions: an immunohistochemical study. Hepatology. 1996; 24(5):1074-1078.

14. Matsumura N, Yamamoto M, Aruga A, Takasaki K, Nakano M. Correlation between expression of
MUC1 core protein and outcome after surgery in mass-forming intrahepatic cholangiocarcinoma. Cancer. 2002;94(6):1770-1776.

15. Settakorn J, Kaewpila N, Burns GF, Leong AS. FAT, E-cadherin, beta catenin, HER 2/neu, Ki67 immuno-expression, and histological grade in intrahepatic cholangiocarcinoma. J Clin Pathol. 2005;58(12):1249-1254.

16. $\mathrm{Li} \mathrm{H}$, et al. In vivo genome editing restores haemostasis in a mouse model of haemophilia. Nature. 2011;475(7355):217-221.

17. Furuyama K, et al. Continuous cell supply from a Sox9-expressing progenitor zone in adult liver, exocrine pancreas and intestine. Nat Genet. 2011; 43(1):34-41.

18. Shiojiri N. Development and differentiation of bile ducts in the mammalian liver. Microsc Res Tech. 1997;39(4):328-335.

19. Aishima S, et al. p62+ Hyaline inclusions in intrahepatic cholangiocarcinoma associated with viral hepatitis or alcoholic liver disease. Am J Clin Pathol. 2010;134(3):457-465.

20. Srinivas $S$, et al. Cre reporter strains produced by targeted insertion of EYFP and ECFP into the ROSA26 locus. BMC Dev Biol. 2001;1:4. 